\title{
Prevalence, Use Behaviors, and Preferences among Users of Heated Tobacco Products: Findings from the 2018 ITC Japan Survey
}

\author{
Edward Sutanto ${ }^{1}{ }^{\circledR}$, Connor Miller ${ }^{1}$, Danielle M. Smith ${ }^{1}$, Richard J. O'Connor ${ }^{1}$, \\ Anne C. K. Quah ${ }^{2}$, K. Michael Cummings ${ }^{3}{ }^{(D}$, Steve $X u^{2}$, Geoffrey T. Fong ${ }^{2,4}$, Andrew Hyland ${ }^{1}$, \\ Janine Ouimet ${ }^{2}$, Itsuro Yoshimi ${ }^{5}$, Yumiko Mochizuki ${ }^{6}$, Takahiro Tabuchi ${ }^{7}{ }^{\circledR}$ and \\ Maciej L. Goniewicz 1,*(D) \\ 1 Division of Cancer Prevention and Population Sciences, Department of Health Behaviors, Roswell Park \\ Comprehensive Cancer Center, Buffalo, NY 14263, USA; edward.sutanto@roswellpark.org (E.S.); \\ Connor.Miller@RoswellPark.org (C.M.); Danielle.Smith@RoswellPark.org (D.M.S.); \\ richard.oconnor@roswellpark.org (R.J.O.); Andrew.Hyland@RoswellPark.org (A.H.) \\ 2 Department of Psychology, University of Waterloo, Waterloo, ON N2L 3G1, Canada; \\ ackquah@uwaterloo.ca (A.C.K.Q.); s4xu@uwaterloo.ca (S.X.); geoffrey.fong@uwaterloo.ca (G.T.F.); \\ j2ouimet@uwaterloo.ca (J.O.) \\ 3 Department of Psychiatry \& Behavioral Sciences, Medical University of South Carolina, \\ Charleston, SC 29425, USA; cummingk@musc.edu \\ 4 Ontario Institute for Cancer Research, Toronto, ON M5G 0A3, Canada \\ 5 Division of Tobacco Policy Research, National Cancer Center Japan, Tokyo 104-0045, Japan; \\ iyoshimi@ncc.go.jp \\ 6 Japan Cancer Society, Tokyo 100-0006, Japan; mochizuki@jcancer.jp \\ 7 Cancer Control Center, Osaka International Cancer Institute, Osaka 537-8511, Japan; tabuchitak@gmail.com \\ * Correspondence: maciej.goniewicz@roswellpark.org
}

Received: 26 October 2019; Accepted: 18 November 2019; Published: 21 November 2019

\begin{abstract}
Heated tobacco products (HTPs), such as IQOS, glo, and Ploom TECH, with a variety of flavored tobacco-containing inserts, have reportedly achieved a significant market share in Japan. We analyzed data from Wave 1 of the ITC Japan Survey, a nationally representative web survey conducted in February to March 2018 among 4684 adult participants to estimate the prevalence of HTP use, describe characteristics of HTP users, and explore user preferences for HTP device and flavor. The overall prevalence of monthly HTP use was $2.7 \%$ (1.7\% daily use). Virtually all HTP users were current cigarette smokers $(67.8 \%)$ or former smokers $(25.0 \%)$; only $1.0 \%$ of HTP users were never smokers. Among HTP users, IQOS was the most frequently reported brand used (64.5\%), and menthol was the most common flavor reported (41.5\%). IQOS was used more by younger respondents and those who reported daily use, while Ploom TECH was more popular among older respondents and non-daily HTP users. This is one of the first non-industry funded studies to explore the use of HTPs in Japan.
\end{abstract}

Keywords: heated tobacco products; heat-not-burn; modified risk tobacco products; flavored tobacco

\section{Introduction}

Heated tobacco products (HTPs) are devices that heat processed tobacco rather than burn the tobacco directly in order to generate a nicotine aerosol for inhalation. Different HTP devices use different heating sources, including electric energy via a battery or carbon tip that is lit with a match or lighter. The HTP system has three common components: (1) an insert (such as stick, capsule, and 
pods) that contains the processed tobacco, (2) a way to heat the tobacco (i.e., battery or carbon tip), and (3) a charger for electrically heated devices [1]. While not an entirely new concept, HTPs have been around for 30 years, with the introduction of Premier by R. J. Reynolds Tobacco Company in 1988 and Accord by Philip Morris (PMI) in 1997 [2,3]. More recently, HTPs have been marketed and promoted by the cigarette companies as safer alternative to combustible cigarettes [4]. Alongside these marketing campaigns, awareness of HTPs has reached significant levels in many countries from 2015 to 2017 (e.g., 48.0\% of Japanese adults [5], 38.1\% of South Korean young adults [6], 19.5\% of Italian adults [7], and 9.3\% of adults in Great Britain had heard of HTPs [1]). In the United States (US), two studies have suggested considerable awareness of HTPs among the adult population, varying from $5.2 \%$ to $12.4 \%$ in 2016 to $2017[8,9]$.

Due in part to lenient national tobacco control measures [10] and the prohibition of electronic nicotine delivery system (ENDS) marketing [11], Japan has been a testing ground for HTPs. IQOS, marketed by PMI, was first introduced in 2014, followed in 2016 by the launches of Ploom TECH by Japan Tobacco International (JTI) and glo by British American Tobacco (BAT) [11]. According to market analyst reports, Japan has the most developed HTP market of all countries worldwide, accounting for $85 \%$ of HTP sales in 2018 [12]. This status has been further cemented by the subsequent release of newer generation HTPs and flavor varieties for tobacco-containing inserts in the Japanese market (Table 1).

PMI has claimed that IQOS attained $15.5 \%$ of the overall tobacco market after just four years of its introduction [12]. While the prevalence of cigarette smoking in Japan steadily decreased over the past decade [17], the introduction of HTPs has raised concerns in achieving further tobacco control progress. HTPs might attract tobacco-naïve individuals, especially youth, with their high-tech appearance and reduced risk claims, both of which align with Japanese cultural norms around cleanliness and respect for others [18-20]. On the other hand, if a significant proportion of cigarette smokers switched to HTPs completely, the associated decline in smoking prevalence may confer substantial benefit towards the public's health. HTPs promoted by the three major tobacco companies (PMI, JTI, and BAT) may employ a variety of marketing strategies to appeal to different sociodemographic user groups. Indeed, differentiated marketing strategies for cigarette smoking have disproportionately appealed to population subgroups, including youth and young adults [21-23], women [23], minorities [24,25], and health-concerned smokers [21].

Most of the data available on the use of HTPs in Japan has come from manufacturers and market analysts. Given the apparent popularity of HTPs in Japan, we felt it was important to independently assess the prevalence and characteristics of users. In this paper, we describe sociodemographic characteristics, product use patterns, device brand and flavor preferences among users of HTPs in Japan, including never smokers, former smokers, and current smokers (dual users). 
Table 1. Characteristics of three major heated tobacco products in Japan.

\begin{tabular}{|c|c|c|c|}
\hline & IQOS & Glo & Ploom TECH \\
\hline \multicolumn{4}{|l|}{ Device picture } \\
\hline Manufacturer & Philip Morris International (PMI) & British American Tobacco (BAT) & Japan Tobacco International (JTI) \\
\hline First launched & November 2014 & December 2016 & March 2016 \\
\hline Type of tobacco inserts & Stick & Stick & Capsule \\
\hline Device generations & $\begin{array}{c}\text { 1st: IQOS } \\
\text { 2nd: IQOS 2.4 } \\
\text { 3rd: IQOS } 3 \text { and IQOS } 3 \text { Multi }\end{array}$ & $\begin{array}{l}\text { 1st: glo } \\
\text { 2nd: glo Series } 2 \text { and glo Series } 2 \text { Mini }\end{array}$ & $\begin{array}{c}\text { 1st: Ploom TECH } \\
\text { 2nd: Ploom TECH+ and Ploom S * }\end{array}$ \\
\hline Brand name of inserts & Marlboro Heatsticks & Kent Neostick & Mevius for Ploom TECH \\
\hline Flavor variety of inserts & $\begin{array}{l}\text { Balanced, Menthol, Mint, Purple } \\
\text { Menthol, Regular, Smooth }\end{array}$ & $\begin{array}{l}\text { Bright Tobacco, Citrus Fresh, Dark Fresh, } \\
\text { Fresh Mix, Intense Fresh, Refreshing } \\
\text { Menthol, Regular, Rich Tobacco, Smooth } \\
\text { Fresh, Spark Fresh, Strong Menthol }\end{array}$ & $\begin{array}{c}\text { Brown Aroma, Cooler Green, Cooler Purple, } \\
\text { Red Cooler, Regular }\end{array}$ \\
\hline Price $^{\dagger}$ & $\begin{array}{c}\text { IQOS 2.4: } ¥ 7980[13] \\
\text { IQOS 3 Multi: } ¥ 8980[14] \\
\text { Marlboro Heatsticks: } ¥ 500 \text { [12] }\end{array}$ & $\begin{array}{c}\text { glo: } ¥ 2980[13] \\
\text { glo Series 2: } ¥ 2980[14] \\
\text { glo Series 2 Mini: } ¥ 3980 \text { [14] } \\
\text { Kent Neostick: } ¥ 420 \text { [15] }\end{array}$ & $\begin{array}{c}\text { Ploom TECH: } ¥ 2980[14] \\
\text { Ploom TECH+: } ¥ 4980[14] \\
\text { Ploom S: } ¥ 7980[14] \\
\text { Mevius for Ploom TECH: } ¥ 490 \text { [16] }\end{array}$ \\
\hline
\end{tabular}

* Ploom S uses sticks instead of capsules. ${ }^{\dagger}$ For comparison, the price of combustible cigarettes is approximately $¥ 500$ [16]. 


\section{Materials and Methods}

\subsection{Data Source}

Data were from the Wave 1 International Tobacco Control (ITC) Japan Survey, a web-based survey administered by Rakuten Insight (https://insight.rakuten.com/) and collected from February to March 2018. The sampling frame of the survey was an existing Rakuten Insight panel that was nationally representative of Japanese cigarette smokers, HTP users, and non-users. Further quotas based on the region of residence, sex, and age, were applied to ensure the final sample of the survey was proportional to stratum sizes based on Japan census data. Adult residents of Japan (aged 20 (the minimum purchasing age) and older, $\mathrm{n}=4684$ ) were sampled as participants of this study. Participants completed an online survey, consisting of questions on HTP and cigarette use, and demographic measures, after eligibility screening. Participants were compensated with a standard Rakuten Insight incentive (points that can be redeemed as cash or merchandise) for a 35-min survey. Both survey procedures and materials were approved by the Office of Research Ethics University of Waterloo.

\subsection{Measures}

\subsubsection{Sociodemographic Variables}

Sociodemographic variables recorded in this study were age, gender, income, and education. Age was categorized into 20-29, 30-39, 40-59, and 60 and older. Participants reported their gender as male or female. Based on Japan's 2016 National Health and Nutrition Survey, annual household income (in Japanese Yen) was defined as follows: low (4,000,000 or less), moderate $(4,000,001-6,000,000)$, high (more than 6,000,000), and refused/do not know [26]. Responses for education were classified as follows: low (junior high school/vocational school/high school), moderate (junior college/technical college), high (undergraduate/postgraduate), and other/refused/do not know.

\subsubsection{Patterns of HTP Use and Cigarette Smoking}

Participants' HTP use status was assessed by the following question: "How often, if at all, do you currently use heat-not-burn products? These include products such as IQOS, Ploom TECH, and glo". Those who answered "daily", "less than daily, but at least once a week", and "less than weekly, but at least once a month" were considered current HTP users. Details of the survey weighting for "less than weekly, but at least once a month" HTP users are provided in Appendix A. Similarly, to assess cigarette smoking status, the following question was asked: "How often, if at all, do you currently smoke cigarettes, including both factory-made and hand-rolled cigarettes?". Participants who responded "daily", "less than daily, but at least once a week", and "less than weekly, but at least once a month" were considered current cigarette smokers. Those who responded "Not at all; I have quit smoking completely" were considered former smokers; while those who responded "Not at all; I have never been a smoker" were considered never smokers. Exclusive users of HTPs or combustible cigarettes were defined as participants who only use either one of the two products and dual users as participants who use both products at least monthly.

Time-to-first HTP use was examined using the following questions: "How soon after waking do you usually draw your first puff on your heat-not-burn product?" for daily HTP users and "On days that you use heat-not-burn products, how soon after waking do you usually draw your first puff?" for weekly and monthly HTP users. Responses were classified into $5 \mathrm{~min}$ or less, 6-30 min, 31-60 min, more than $60 \mathrm{~min}$, and refuse/do not know.

The number of tobacco-containing inserts per day was only assessed for daily and weekly HTP users. For daily HTP users, tobacco-containing inserts per day was assessed by the following question: "On a typical day, how many heat-not-burn tobacco sticks or capsules do you usually use?". For weekly HTP users, we asked the following question: "On a typical week, how many heat-not-burn tobacco 
sticks or capsules do you usually use?" and divided the response by seven to get the number of tobacco-containing inserts per day.

\subsubsection{HTP Device Brand Preferences}

Device brand preference was assessed using the following question: "Thinking now about the actual heating device that is used with tobacco sticks or capsules. Which of the following devices have you ever used?". The options following the questions were "IQOS", "glo", "Ploom Tech", and "other (specify)". If participants selected more than one device brand, an additional question was asked: "Which heating device do you use most?" with the same options being given for answer.

Reason for choosing device brand was examined using the following question: "In choosing your heating device, was your choice based on any of the following?", and the participants were instructed to respond for each of the following: (a) It may not be as bad for my health; (b) The price; (c) The taste of the sticks or capsules; (d) The design of the heating device, charging tools, etc. (Please only think about the heating/charging device, not sticks or capsules); (e) The time it takes the device to heat the sticks or capsules; (f) The advertising; (g) It is more available; (h) My friends use this product; and (i) People in the media or other public figures use this product. In each statement, the response options were "yes", "no", "refused", and "do not know" thus participants could select multiple reasons for choosing a device brand.

\subsubsection{HTP Flavor Preferences}

Flavor preference was evaluated through the following question: "What variety of flavor of tobacco sticks or capsules do you usually use?". In addition to "refused", "other (specify)", "do not know", we initially provided 22 options of tobacco sticks and capsules that were available for purchase in the Japanese market. We re-classified those flavors to tobacco, menthol, mentholated fruity, coffee, and refused/do not know. Further details of the classification of the flavor option for tobacco sticks and capsules are provided in Appendix B.

\subsection{Statistical Analysis}

We presented descriptive statistics of the study population in weighted percentage and $95 \%$ confidence interval (CI). Rao-Scott chi-square tests were conducted to both examine the difference between general characteristics of the study population and HTP use by smoking status, along with device brand preference. Normality for the number of tobacco-containing inserts per day was tested with the Shapiro-Wilk test. Due to the non-normal distribution of this variable, a Kruskal-Wallis test was employed to examine differences in the number of tobacco-containing inserts per day between each smoking status or device brand. Multiple comparisons were addressed by using Dunn's test to produce adjusted $p$-values. Weighted multiple logistic regression analyses were performed to investigate the association between the use of each device brand and demographic characteristics, adjusting for frequency of HTP use. All statistical analyses were performed using svy commands in Stata SE version 14.2 (StataCorp, College Station, TX, USA). All tests were two-tailed and considered significant at $p<0.05$. Further details on the weighting and sampling strategy are provided in the ITC Japan Survey Technical Report (https://itcproject.org/files/JP1-1.5_Technical_Report_5June2019.pdf).

\section{Results}

\subsection{Prevalence of HTP Use and User Characteristics}

Table 2 shows the characteristics of the study population stratified by smoking status. The overall prevalence of HTP use was $2.7 \%$ (95\% CI: $2.4-3.0 \%$ ), while the prevalence of exclusive HTP use was $0.9 \%$ (95\% CI: $0.7-1.1 \%$ ). Among HTP users, $67.8 \%$ (95\% CI: $63.1-72.2 \%), 25.0 \%$ (95\% CI: 20.8-29.8\%), and $1.0 \%$ (95\% CI: 0.4-2.0\%) were current smokers, former smokers, and never smokers, respectively. A greater proportion of HTP users were male, aged 40-59, and belonged to the high-income 
$(>6,000,000$ Japanese Yen) group. The majority of HTP users who were current smokers and former smokers fell within the high education category (i.e., undergraduate or postgraduate degree).

\subsection{Patterns of HTP Use and Cigarette Smoking}

The majority of HTP users (63.4\%; 95\% CI: 58.9-67.6\%) reported using HTPs on a daily basis, with daily use being more common among exclusive HTP users (88.3\%; 95\% CI: 80.5-93.2\%). While the majority of HTP users among the three smoking status categories reported daily use of HTP (current smokers: 51.5\%, 95\% CI: 46.7-56.3\%; former smokers: 86.9\%, 95\% CI: 77.2-92.9\%; and never smokers: $100 \%)$, current smokers had the highest percentage for weekly and monthly use of HTP $(19.1 \%, 95 \%$ CI: 16.1-22.5\%; and 29.4\%, 95\% CI: 24.4-34.9\%, respectively). HTP users most commonly reported that they use HTP 6-30 min after waking up (current HTP users: 33.8\%, 95\% CI: 29.7-38.1\%; and exclusive HTP users: $40.9 \%$, 95\% CI: $32.2-50.1 \%$ ).

The number of tobacco-containing inserts per day (median, (interquartile range)) for all HTP users and exclusive HTP users was 10.0 (2.8-15.0) and 10.0 (5.0-18.0). While the number of tobacco-containing inserts per day was not significantly different for HTP users who were former smokers and never smokers (former smokers: 10.0 (5.0-18.0) and never smokers: 15.0 (3.0-20.0)), it was significantly lower for those who also concurrently smoked cigarettes (7.0 (1.4-15.0); both $p<0.05)$. 
Table 2. Prevalence of HTP use and user characteristics.

\begin{tabular}{|c|c|c|c|c|c|c|}
\hline \multirow{3}{*}{ Variable } & \multirow{3}{*}{ Category } & \multirow{2}{*}{$\begin{array}{l}\text { Current HTP User } \\
\quad(\mathrm{n}=859)\end{array}$} & \multirow{2}{*}{$\begin{array}{l}\text { Exclusive HTP User } \\
\qquad(\mathrm{n}=170)\end{array}$} & \multicolumn{3}{|c|}{ HTP User by Smoking Status * } \\
\hline & & & & $\begin{array}{l}\text { Current Smokers } \\
(n=689)\end{array}$ & $\begin{array}{c}\text { Former Smokers } \\
\quad(\mathrm{n}=115)\end{array}$ & $\begin{array}{c}\text { Never Smokers } \\
\quad(n=7)\end{array}$ \\
\hline & & \multicolumn{5}{|c|}{ Weighted \% (95\% Confidence Interval) } \\
\hline \multicolumn{2}{|c|}{ Prevalence } & $2.7(2.4-3.0)$ & $0.9(0.7-1.1)$ & $1.8(1.6-2.1)$ & $0.7(0.5-0.9)$ & $0.02(0.01-0.06)$ \\
\hline \multicolumn{7}{|c|}{ Demographics } \\
\hline \multirow{2}{*}{ Gender } & Male & $76.0(72.0-79.7)$ & $71.8(63.5-78.8)$ & $78.1(73.3-82.2)$ & $72.8(62.9-80.8)$ & $62.7(23.0-90.4)$ \\
\hline & Female & $24.0(20.3-28.0)$ & $28.2(21.2-36.4)$ & $21.9(17.8-26.7)$ & $27.2(19.1-37.1)$ & $37.3(9.5-77.0)$ \\
\hline & & & & \multicolumn{3}{|c|}{$\mathrm{F}(1.93,1551.85)=0.91 ; p=0.400^{\dagger}$} \\
\hline \multirow{5}{*}{ Age (years) } & $20-29$ & $16.6(13.2-20.7)$ & $12.9(7.1-22.3)$ & $18.4(14.5-22.9)$ & $14.8(7.7-26.6)$ & - \\
\hline & $30-39$ & $26.1(22.6-29.8)$ & $22.6(16.2-30.5)$ & $27.7(23.8-32.0)$ & $21.8(14.4-31.4)$ & $47.6(15.6-81.7)$ \\
\hline & $40-59$ & $44.0(39.7-48.3)$ & $55.7(46.4-64.6)$ & $38.4(34.0-43.0)$ & $54.4(43.3-65.2)$ & $52.4(18.3-84.4)$ \\
\hline & 60 and older & $13.3(10.6-16.7)$ & $8.8(4.7-15.8)$ & $15.5(12.2-19.5)$ & $9.0(4.3-17.9)$ & - \\
\hline & & & & \multicolumn{3}{|c|}{$\mathrm{F}(5.23,4200.06)=1.72 ; p=0.122$} \\
\hline \multirow{5}{*}{ Annual Household Income } & Low & $15.8(13.0-19.0)$ & $14.3(9.3-21.3)$ & $16.5(13.4-20.1)$ & $14.9(8.9-23.7)$ & $11.9(1.6-53.5)$ \\
\hline & Moderate & $22.6(18.2-26.3)$ & $19.3(13.2-27.2)$ & $24.1(20.3-28.4)$ & $19.6(12.5-29.4)$ & $10.3(1.3-49.5)$ \\
\hline & High & $51.0(46.6-55.3)$ & $60.6(51.6-68.9)$ & $46.5(41.8-51.2)$ & $61.3(50.4-71.1)$ & $60.4(24.0-88.0)$ \\
\hline & Refused/Do not know & $10.6(7.9-14.1)$ & $5.8(3.1-10.6)$ & $12.9(9.4-17.6)$ & $4.2(1.8-9.8)$ & $17.3(2.4-64.1)$ \\
\hline & & & & \multicolumn{3}{|c|}{$\mathrm{F}(5.42,4351.58)=2.18 ; p=0.048$} \\
\hline \multirow{5}{*}{ Education } & Low & $25.9(22.6-29.5)$ & $24.6(18.3-32.2)$ & $26.5(22.9-30.5)$ & $21.9(15.1-30.8)$ & - \\
\hline & Moderate & $21.4(17.8-25.3)$ & $22.1(16.3-29.1)$ & $21.0(16.7-26.0)$ & $18.7(12.6-26.7)$ & $65.0(28.2-89.7)$ \\
\hline & High & $51.9(47.6-56.3)$ & $52.9(43.9-61.7)$ & $51.5(46.7-56.2)$ & $58.9(48.3-68.6)$ & $35.0(10.2-71.8)$ \\
\hline & $\begin{array}{l}\text { Other/Refused/Do not } \\
\text { know }\end{array}$ & $0.8(0.4-1.7)$ & $0.4(0.1-2.9)$ & $1.0(0.4-2.3)$ & $0.5(0.1-3.8)$ & - \\
\hline & & & & \multicolumn{3}{|c|}{$\mathrm{F}(5.53,4439.27)=1.63 ; p=0.140$} \\
\hline
\end{tabular}


Table 2. Cont

\begin{tabular}{|c|c|c|c|c|c|c|}
\hline \multirow{3}{*}{ Variable } & \multirow{3}{*}{ Category } & \multirow{2}{*}{$\begin{array}{l}\text { Current HTP User } \\
\quad(\mathrm{n}=859)\end{array}$} & \multirow{2}{*}{$\begin{array}{l}\text { Exclusive HTP User } \\
\qquad(\mathrm{n}=170)\end{array}$} & \multicolumn{3}{|c|}{ HTP User by Smoking Status * } \\
\hline & & & & $\begin{array}{l}\text { Current Smokers } \\
(\mathrm{n}=689)\end{array}$ & $\begin{array}{c}\text { Former Smokers } \\
\quad(\mathrm{n}=115)\end{array}$ & $\begin{array}{c}\text { Never Smokers } \\
\quad(n=7)\end{array}$ \\
\hline & & \multicolumn{5}{|c|}{ Weighted \% (95\% Confidence Interval) } \\
\hline \multicolumn{7}{|c|}{ Product Use Pattern } \\
\hline & Daily & $63.4(58.9-67.6)$ & $88.3(80.5-93.2)$ & $51.5(46.7-56.3)$ & $86.9(77.2-92.9)$ & 100.0 \\
\hline Frequency of HTP Use & Weekly & $16.1(13.5-19.1)$ & $9.9(5.7-16.7)$ & $19.1(16.1-22.5)$ & $10.8(5.8-19.3)$ & - \\
\hline & Monthly & $20.5(16.7-24.9)$ & $1.8(0.2-11.6)$ & $29.4(24.4-34.9)$ & $2.3(0.3-14.6)$ & - \\
\hline & & & & \multicolumn{3}{|c|}{$\mathrm{F}(3.03,2432.26)=9.56 ; p<0.001$} \\
\hline \multirow{6}{*}{ Time to first HTP use } & 5 min or less & $15.4(12.8-18.5)$ & $17.5(12.0-24.8)$ & $14.4(11.7-17.6)$ & $15.1(9.1-23.9)$ & $42.0(13.0-77.8)$ \\
\hline & 6-30 min & $33.8(29.7-38.1)$ & $40.9(32.2-50.1)$ & $30.4(62.0-35.1)$ & $42.6(32.1-53.7)$ & $25.4(3.9-74.1)$ \\
\hline & $31-60 \mathrm{~min}$ & $15.5(12.5-19.0)$ & $18.9(12.5-27.6)$ & $13.9(11.0-17.3)$ & $19.8(12.2-30.5)$ & $10.4(1.3-49.5)$ \\
\hline & More than $60 \mathrm{~min}$ & $32.8(28.9-37.1)$ & $20.9(14.2-29.6)$ & $38.5(33.9-43.3)$ & $20.2(12.5-31.1)$ & $22.2(5.1-60.4)$ \\
\hline & Refused/Do not know & $2.5(1.3-4.6)$ & $1.8(0.2-11.6)$ & $2.8(1.6-5.0)$ & $2.3(0.3-14.6)$ & - \\
\hline & & & & \multicolumn{3}{|c|}{$\mathrm{F}(6.27,5038.00)=1.91 ; p=0.071$} \\
\hline Tobacco-containir & nserts per day $\ddagger$ & $10.0(2.8-15.0)$ & $10.0(5.0-18.0)$ & $7(1.4-15.0)$ & $10.0(5.0-18.0)$ & $15.0(3.0-20.0)$ \\
\hline \multicolumn{7}{|c|}{ Device Brand Preferences } \\
\hline \multirow{4}{*}{ Device Brand } & IQOS & $64.5(60.3-68.6)$ & $74.0(65.3-81.1)$ & $60.1(55.3-64.7)$ & $74.8(64.1-83.2)$ & 100.0 \\
\hline & glo & $14.4(11.7-17.5)$ & $8.2(4.6-14.2)$ & $17.2(13.9-21.2)$ & $8.9(4.6-16.5)$ & - \\
\hline & Ploom TECH & $21.1(17.8-24.8)$ & $17.8(11.8-25.9)$ & $22.7(18.9-26.9)$ & $16.3(9.5-26.4)$ & - \\
\hline & & & & \multicolumn{3}{|c|}{$\mathrm{F}(3.68,2921.15)=2.69 ; p=0.033$} \\
\hline \multicolumn{7}{|c|}{ Flavor Preferences } \\
\hline \multirow{5}{*}{ Flavor } & Tobacco & $33.7(29.8-37.7)$ & $29.3(21.8-38.1)$ & $35.7(31.5-40.2)$ & $26.8(18.1-37.6)$ & $35.0(10.2-71.8)$ \\
\hline & Menthol & $41.5(37.2-45.9)$ & $47.5(38.5-56.7)$ & $38.6(34.2-43.3)$ & $49.2(38.4-60.2)$ & $65.0(28.2-89.7)$ \\
\hline & Mentholated fruity $\S$ & $20.0(16.4-24.1)$ & $20.3(13.8-28.9)$ & $19.8(15.7-24.5)$ & $21.0(13.2-31.7)$ & - \\
\hline & Coffee & $3.1(1.9-4.9)$ & $0.9(0.2-3.9)$ & $4.1(2.5-6.7)$ & $0.8(0.1-5.5)$ & - \\
\hline & Refused/Do not know & $1.8(0.9-3.4)$ & $2.0(0.6-6.1)$ & $1.7(0.7-3.7)$ & $2.2(0.6-7.8)$ & - \\
\hline
\end{tabular}

* Discrepancy in total due to a small proportion of occasional smokers (less than monthly). ${ }^{\dagger}$ Rao-Scott chi-square tests accounted the complex survey design. Resulting test stats were design-based $\mathrm{F}$ with respective degrees of freedom for each comparison. ${ }^{\ddagger}$ Number shown are median (interquartile range). ${ }^{\S}$ Including mentholated vanilla. 
Table 3 presents HTP use patterns and flavor preferences according to device brand preference. Although daily use was the most prevalent pattern across users of three device brands (IQOS: 69.6\%, 95\% CI: 63.9-74.7\%; glo: 60.1\%, 95\% CI: 48.2-70.9\%; and Ploom TECH: 49.1\%, 95\% CI: 39.9-58.3\%), Ploom TECH users had the highest percentage of weekly and monthly HTP users compared to the other two device brands (weekly: 20.2\%, 95\% CI: 14.6-27.3\%; monthly: 30.7\%, 95\% CI: $22.4-40.5 \%$ ). IQOS users most frequently reported 6-30 min for time-to-first HTP use (36.6\%, 95\% CI: $31.3-42.3 \%)$, while glo and Ploom TECH users most often reported more than $60 \mathrm{~min}$ (40.3\%, 95\% CI: 29.6-52.0\%; and $46.5 \%$, 95\% CI: 37.4-55.8\%, respectively). The number of tobacco-containing inserts per day for IQOS, glo, and Ploom TECH users were 10.0 (5.0-18.0), 10.0 (5.0-18.0), and 1.0 (0.7-5.0), respectively. There was a statistically significant difference for the number of tobacco-containing inserts per day between Ploom TECH users and IQOS users, along with Ploom TECH and glo users (both $p<0.001$ ).

Table 3. Product use pattern and flavor preference according to device brand preference.

\begin{tabular}{|c|c|c|c|c|c|}
\hline & & \multicolumn{3}{|c|}{ HTP Device Brand } & \multirow{3}{*}{$p$-Value * } \\
\hline & & \multicolumn{3}{|c|}{ Weighted \% (95\% Confidence Interval) } & \\
\hline & & IQO $(n=547)$ & Glo $(n=131)$ & Ploom TECH $(n=172)$ & \\
\hline \multicolumn{6}{|c|}{ Product Use Pattern } \\
\hline \multirow{3}{*}{ Frequency of HTP Use } & Daily & $69.6(63.9-74.7)$ & $60.1(48.2-70.9)$ & $49.1(39.9-58.3)$ & \multirow{3}{*}{0.004} \\
\hline & Weekly & $14.8(11.7-18.7)$ & $16.0(10.3-24.1)$ & $20.2(14.6-27.3)$ & \\
\hline & Monthly & $15.6(11.1-21.3)$ & $23.9(14.0-37.5)$ & $30.7(22.4-40.5)$ & \\
\hline \multirow{5}{*}{ Time to first HTP use } & 5 min or less & $19.2(15.4-23.6)$ & $13.9(8.7-21.4)$ & $6.2(3.4-11.2)$ & \multirow{5}{*}{0.001} \\
\hline & $6-30 \mathrm{~min}$ & $36.6(31.3-42.3)$ & $26.6(18.9-36.1)$ & $29.6(21.5-39.3)$ & \\
\hline & $31-60 \mathrm{~min}$ & $15.8(12.1-20.4)$ & $15.2(8.5-25.7)$ & $16.3(10.3-24.8)$ & \\
\hline & More than $60 \mathrm{~min}$ & $26.5(21.9-31.6)$ & $40.3(29.6-52.0)$ & $46.5(37.4-55.8)$ & \\
\hline & Refused/Do not know & $1.9(0.6-5.4)$ & $4.0(1.3-11.4)$ & $1.4(0.4-4.2)$ & \\
\hline \multicolumn{2}{|c|}{ Tobacco-containing inserts per day $^{\dagger}$} & $10.0(5.0-18.0)$ & $10.0(5.0-15.0)$ & $1.0(0.7-5.0)$ & $<0.001$ \\
\hline \multicolumn{6}{|c|}{ Device Brand } \\
\hline \multirow{9}{*}{$\begin{array}{l}\text { Reason for choosing } \\
\text { specific device brand } \ddagger\end{array}$} & $\begin{array}{l}\text { Perceived reduction in health risk } \\
\text { compared to smoking }\end{array}$ & $64.7(59.2-69.8)$ & $59.2(48.4-69.2)$ & $68.4(59.2-76.4)$ & 0.364 \\
\hline & Price & $18.2(14.0-23.3)$ & $37.2(27.8-47.6)$ & $35.4(26.9-44.9)$ & 0.001 \\
\hline & Taste & $43.3(37.8-49.0)$ & $36.3(27.0-46.7)$ & $48.4(39.2-57.7)$ & 0.223 \\
\hline & Design & $37.6(32.1-43.4)$ & $29.4(20.1-39.4)$ & $39.3(30.7-48.6)$ & 0.768 \\
\hline & Time to heat & $21.7(17.8-26.2)$ & $42.0(31.5-53.3)$ & $54.0(44.6-63.1)$ & $<0.001$ \\
\hline & Advertising & $24.9(20.8-29.5)$ & $23.1(13.6-36.5)$ & $16.9(11.4-24.3)$ & 0.703 \\
\hline & Availability & $56.5(50.7-62.1)$ & $53.2(42.1-64.0)$ & $34.5(26.4-43.6)$ & 0.002 \\
\hline & Used by friends & $68.1(62.8-73.0)$ & $41.4(31.4-52.2)$ & $25.8(18.5-34.8)$ & $<0.001$ \\
\hline & Endorsement in media & $22.9(18.2-28.5)$ & $8.3(4.8-14.1)$ & $5.9(2.9-11.3)$ & $<0.001$ \\
\hline \multicolumn{6}{|c|}{ Flavor } \\
\hline \multirow{5}{*}{ Flavor } & Tobacco & $37.0(32.0-42.3)$ & $28.1(20.5-37.7)$ & $29.2(21.9-37.8)$ & \multirow{5}{*}{$<0.001$} \\
\hline & Menthol & $52.6(47.0-58.2)$ & $51.8(41.0-62.4)$ & - & \\
\hline & Mentholated fruity $\S$ & $9.5(5.9-15.0)$ & $19.0(12.4-28.0)$ & $54.6(45.3-63.5)$ & \\
\hline & Coffee & - & - & $15.0(9.5-22.9)$ & \\
\hline & Refused/Do not know & $0.9(0.3-3.1)$ & $1.1(0.3-4.5)$ & $1.2(0.4-3.0)$ & \\
\hline
\end{tabular}

* Rao-Scott chi-square tests were used to account for the complex survey design. ${ }^{\dagger}$ Number shown are median (interquartile range). $\ddagger$ Percentage shown for those who responded yes to the statement. With other response options, added up to $100 \%$. § Including mentholated vanilla.

\subsection{Device Brand Preference}

IQOS was the most prevalent device among current HTP users (64.5\%, 95\% CI: 60.3-68.6\%), followed by Ploom TECH (21.1\%, 95\% CI: $17.8-24.8 \%)$, and glo (14.4\%, 95\% CI: $11.7-17.5 \%)$. Among exclusive HTP users, the pattern of device brand preference was similar (IQOS: $74.0 \%$, 95\% CI: 65.3-81.1\%; Ploom TECH: 17.8\%, 95\% CI: 11.8-25.9\%; and glo: 8.2\%, 95\% CI: 4.6-14.2\%). There was a statistically significant association between device brand preference and HTP use by smoking status. IQOS was the most common HTP device brand reported by current smokers, former smokers, and never smokers (current smokers: $60.1 \%$, 95\% CI: 55.3-64.7\%; former smokers: $74.8 \%$, 95\% CI: 64.1-83.2\%; and never smokers: $100 \%$ ). 
Figure 1 shows the device brand preference by various demographic characteristics. There was an association between device brand preference and age group (Figure 1A). While IQOS was the most preferred device across all age groups, its use tended to be lower among older age groups compared to those aged 20 to 29. Conversely, Ploom TECH use was higher among older age groups, with $41.5 \%$ (95\% CI: 29.9-54.2\%) of those aged 60 and older using it (Figure 1A). IQOS was the most preferred device among both males and females (Figure 1B). IQOS also was the most preferred device brand across the income and education categories, while glo was the least preferred device brand (Figure 1C,D, respectively). 

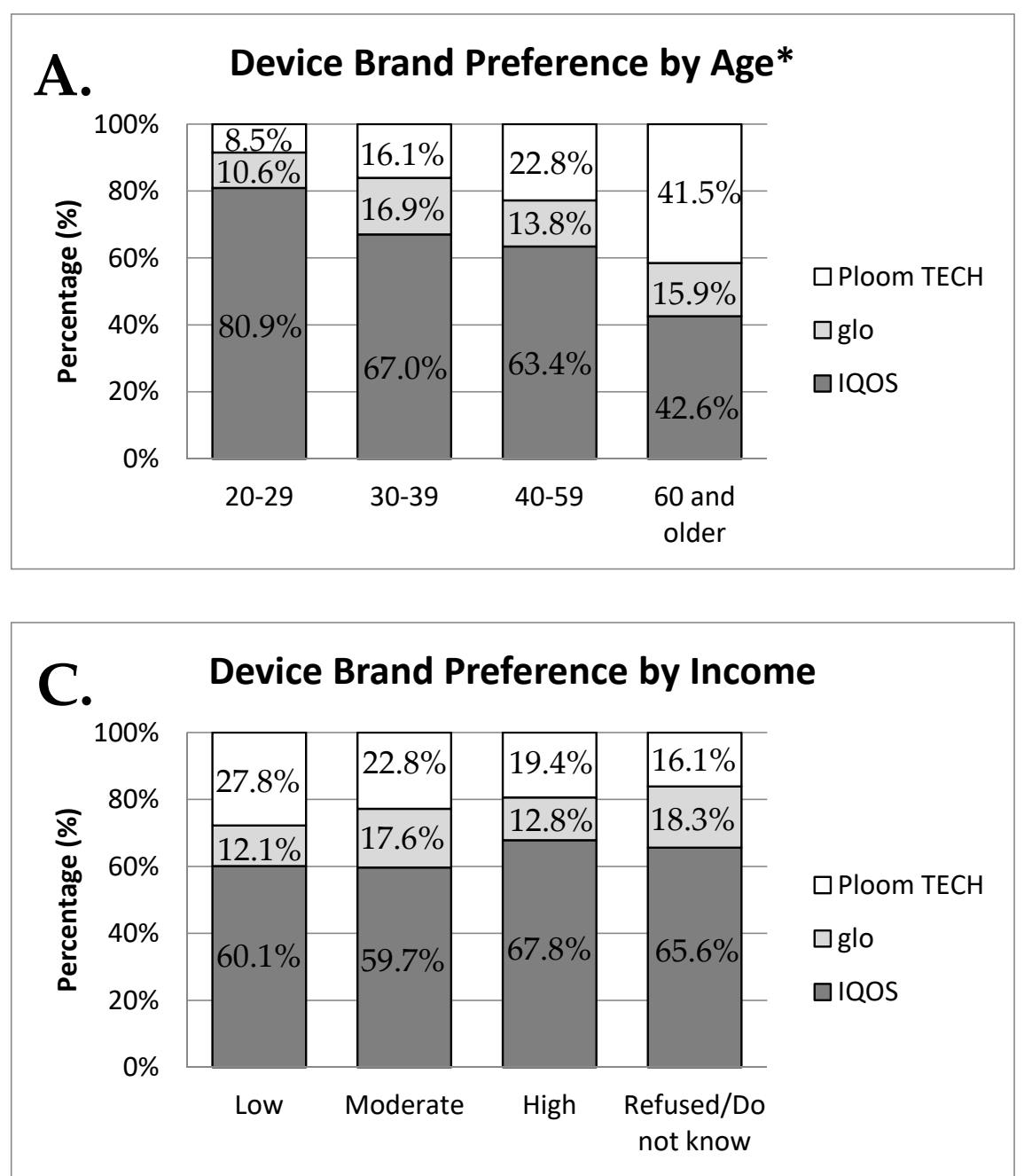

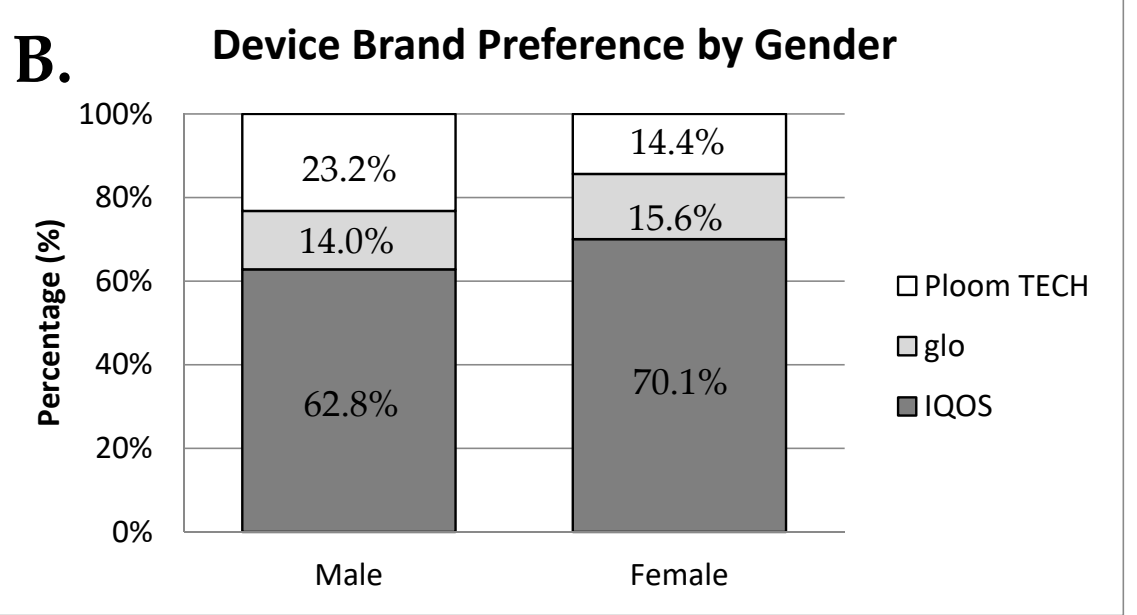

\section{Device Brand Preference by Education}

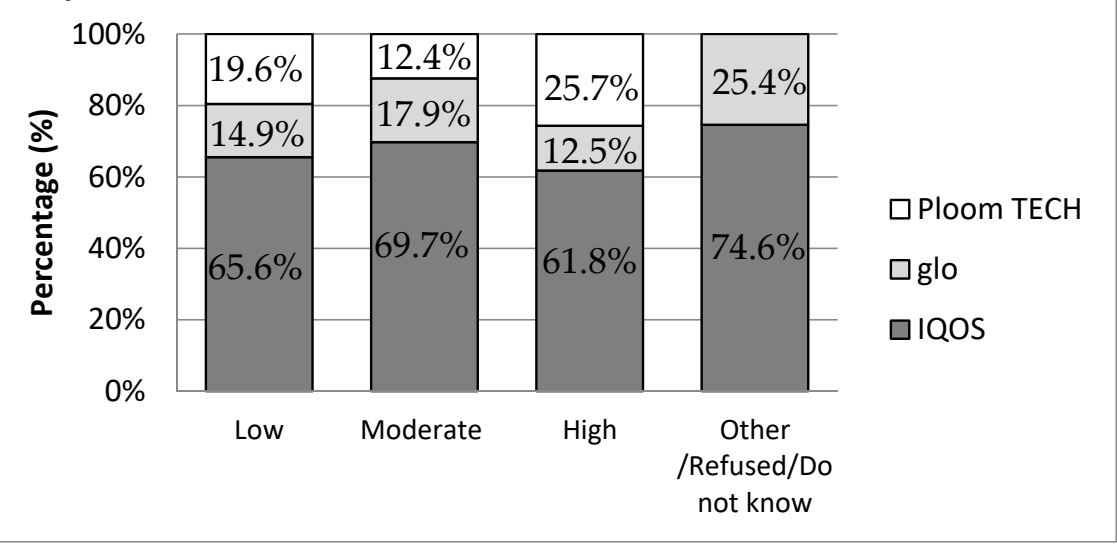

Figure 1. HTP device brand preference by various demographic characteristics. (A) Age; (B) gender; (C) annual household income; (D) education. The values represent a weighted percentage. ${ }^{*} p<0.001$ (Rao-Scott chi-square tests). 
The association between sociodemographic characteristics and each device brand among current HTP users (adjusted for frequency of HTP use) can be seen in Table 4. Compared to those aged 20-29, those aged 30-39, 40-59, and aged 60 and older had lower odds (adjusted odds ratios (95\% CI)) of using IQOS (30-39: $0.46(0.24-0.86), 40-59: 0.36(0.20-0.66)$, and aged 60 and older: $0.20(0.09-0.43))$ but higher odds of using Ploom TECH (40-59: 3.69 (1.61-8.92) and aged 60 and older: 6.32 (2.44-17.33)). No other sociodemographic characteristics were noted to be consistently associated with each device brand use. Compared to daily HTP users, those who use HTP weekly and monthly had lower odds of using IQOS (weekly: 0.61 (0.39-0.95); monthly: 0.49 (0.29-0.83)). In contrast, weekly and monthly HTP users had higher odds of using Ploom TECH (weekly: 1.89 (1.12-3.17); monthly: 2.12 (1.19-3.76)).

Table 4. Multiple logistic regression examining sociodemographic characteristics by device brand preference.

\begin{tabular}{|c|c|c|c|c|}
\hline & & IQOS * & Glo $^{+}$ & Ploom TECH $\ddagger$ \\
\hline & & aOR $(95 \% \mathrm{CI})$ & aOR $(95 \% \mathrm{CI})$ & aOR $(95 \% \mathrm{CI})$ \\
\hline \multirow{2}{*}{ Gender } & Male & Ref & Ref & Ref \\
\hline & Female & $1.14(0.73-1.78)$ & $0.96(0.58-1.60)$ & $0.84(0.47-1.51)$ \\
\hline \multirow{4}{*}{ Age (years) } & $20-29$ & Ref & Ref & Ref \\
\hline & $30-39$ & $0.46(0.24-0.86)$ & $1.62(0.78-3.35)$ & $2.26(0.90-5.67)$ \\
\hline & $40-59$ & $0.36(0.20-0.66)$ & $1.35(0.67-2.71)$ & $3.79(1.61-8.92)$ \\
\hline & 60 and older & $0.20(0.09-0.43)$ & $1.53(0.55-4.21)$ & $6.52(2.44-17.44)$ \\
\hline \multirow{4}{*}{ Annual Household Income } & Low & Ref & Ref & Ref \\
\hline & Moderate & $0.95(0.51-1.76)$ & $1.49(0.69-3.18)$ & $0.83(0.39-1.75)$ \\
\hline & High & $1.49(0.85-2.61)$ & $1.10(0.59-2.08)$ & $0.55(0.27-1.11)$ \\
\hline & Refused/Do not know & $1.22(0.58-2.59)$ & $1.48(0.60-3.66)$ & $0.56(0.22-1.46)$ \\
\hline \multirow{4}{*}{ Education } & Low & Ref & Ref & Ref \\
\hline & Moderate & $1.26(0.76-2.09)$ & $1.16(0.61-2.21)$ & $0.58(0.32-1.07)$ \\
\hline & High & $0.84(0.54-1.31)$ & $0.83(0.49-1.44)$ & $1.42(0.83-2.45)$ \\
\hline & Other/Refused/Do not know & $1.28(0.16-9.91)$ & $1.66(0.16-17.18)$ & $-\S$ \\
\hline \multirow{3}{*}{ Frequency of HTP Use } & Daily & Ref & Ref & Ref \\
\hline & Weekly & $0.61(0.39-0.95)$ & $1.08(0.61-1.93)$ & $1.89(1.12-3.17)$ \\
\hline & Monthly & $0.49(0.29-0.83)$ & $1.26(0.62-2.56)$ & $2.12(1.19-3.76)$ \\
\hline
\end{tabular}

* Model 1 compares IQOS to non-IQOS device brands (glo + Ploom TECH). ${ }^{\dagger}$ Model 2 compares glo to non-glo device brands (IQOS + Ploom TECH). ${ }^{\ddagger}$ Model 3 compares Ploom TECH to non-Ploom TECH device brands (IQOS + glo). ${ }^{\S}$ No observation for "Other/Refused/Do not know" was recorded in Ploom TECH users.

The most common reason for choosing IQOS was "used by friend" (68.1\%, 95\% CI: 62.8-73.0\%), while for glo and Ploom TECH it was the "perceived reduction in health risk compared to smoking" (59.2\%, 95\% CI: 48.4-69.2\%; and 68.4\%, 95\% CI: 59.2-76.4\%, respectively). Among the three device brands, there were significant differences for several reasons for choosing a device brand, including price, time-to-heat, availability, used by friend, and endorsement in media (all $p<0.05$ ).

\subsection{Flavor Preference}

Menthol (41.5\%, 95\% CI: 37.2-45.9\%); tobacco (33.7\%, 95\% CI: 29.8-37.7\%), and mentholated fruity (20.0\%, 95\% CI: $16.4-24.1 \%)$ were the most commonly preferred flavors among current HTP users. This flavor preference was also similar to exclusive HTP users (menthol: $47.5 \%$, 95\% CI: 38.5-56.7\%; tobacco: 29.3\%, 95\% CI: 21.8-38.1\%; and mentholated fruity: $20.3 \%$, 95\% CI: $13.8-28.9 \%)$. For HTP users who were current smokers, former smokers, and never smokers, the three most commonly preferred flavors were menthol (current smokers: $38.6 \%, 95 \%$ CI: $34.2-43.3 \%$; former smokers: $49.2 \%$, 95\% CI: 38.4-60.2\%; and never smokers: $65.0 \%, 95 \%$ CI: $28.2-89.7 \%$ ), tobacco (current smokers: $35.7 \%$, 95\% CI: $31.5-40.2 \%$; former smokers: $26.8 \%$, 95\% CI: $18.1-37.6 \%$; and never smokers: $35.0 \%, 95 \%$ CI: 10.2-71.8\%), and mentholated fruity (current smokers: $19.8 \%, 95 \%$ CI: $15.7-24.5 \%$; and former smokers: $21.0 \%, 95 \%$ CI: $13.2-31.7 \%$ ).

Among IQOS and glo users, the most commonly preferred flavors were menthol (IQOS: $52.6 \%$, 95\% CI: 47.0-58.2\%; and glo: 51.8\%, 95\% CI: 41.0-62.4\%), followed by tobacco (IQOS: $37.0 \%$, 95\% CI: 
32.0-42.3\%; and glo: $28.1 \%, 95 \%$ CI: 20.5-37.7\%), and mentholated fruity (IQOS: 9.5\%, 95\% CI 5.9-15.0\%; and glo: $19.0 \%$, 95\% CI: 12.4-28.0\%). As there was no menthol flavor available for Ploom TECH, the most commonly preferred flavors were mentholated fruity $(54.6 \%, 95 \%$ CI: $45.3-63.5)$, tobacco $(29.2 \%$, 95\% CI: $21.9-37.8 \%)$, and coffee (15.0\%, 95\% CI: 9.5-22.9\%).

\section{Discussion}

\subsection{Prevalence and Pattern of HTP Use}

This study adds to the limited body of evidence on HTPs by describing sociodemographic characteristics, product use patterns, device brand and flavor preferences among users of HTPs in Japan, including never smokers, former smokers, and current smokers (dual users). Using a national representative sample of tobacco users and validated survey instruments, we estimated that the overall prevalence of current HTP use among adults in Japan was $2.7 \%$ in 2018 . To our knowledge, only two other independent studies attempted to estimate the prevalence of current HTP use in Japan. Using repeated online surveys, these studies found there was an increase in the prevalence of current HTP use (defined as use in the past 30 days) from 1.3\% in 2015 to $4.7 \%$ in 2017 [5,11]. Both estimates, however, were combined percentages for current HTP use and ENDS use. While the prevalence of current ENDS use might be negligible in Japan due to the ban on the sale of ENDS, our study provides the most recent and more precise estimate of the prevalence of current HTP use. Future studies should also investigate the prevalence and pattern of HTP use among adolescents (aged 13 to 19).

Both independent and tobacco industry-funded studies have reported that the prevalence of cigarette smoking is trending downwards in Japan $[17,27]$. Currently, there are no data on the impact of HTP availability and use on cigarette smoking prevalence. Our data showed that the majority of HTP users are current and former cigarette smokers, with only 1.0\% of HTP users who have never smoked cigarettes. Because of the limited number of participants in our survey that are HTP users who have never smoked cigarettes, we were not able to analyze this group in a detailed manner. As a HTP user is still exposed to harmful constituents [2], the uptake of HTP use among never smokers and former smokers is a crucial data point to continue assessing into the future. While the use of ENDS has previously been associated with a greater likelihood of initiating cigarette smoking among youth and young adults [28,29], it is currently unknown whether HTPs will follow a similar trajectory. Conversely, a national study in the Republic of Korea observed similar concurrent cigarette use patterns among HTP users [30]. High rates of dual use among current cigarette smokers, along with lower average tobacco inserts used per day, and longer time-to-first HTP than former or never smokers in this study suggest that HTPs may often serve as complementary products, rather than substitutes for cigarette smoking. Future longitudinal studies may show whether HTP dual use patterns are similar to those observed in other countries for ENDS, and whether dual HTP-cigarette use serve as a transitional behavioral state toward cigarette smoking cessation. Still, substantial dual use of cigarettes and HTPs warrants skepticism toward industry claims of risk-reduction.

\subsection{Device Brand Preference}

Among current HTP users, we found that IQOS was the most commonly used HTP device brand, followed by Ploom TECH and glo, consistent with prior research [11]. In addition to IQOS being introduced earlier than competitor products, PMI's IQOS marketing strategy emphasizes important values within the Japanese culture, such as cleanliness, exclusivity, and a high-tech appearance [20], which has likely played a role in IQOS's popularity. As new generations of HTPs are likely to come about, we plan to examine trends in device brand popularity, using the subsequent waves of data that will be collected for the ITC Japan Survey.

Among current HTP users, the odds of using IQOS were higher among young adults, while the opposite was true for Ploom TECH users, indicating a highly important sociodemographic difference between the two HTP brands. This contrasts with the PMI-funded study that reported IQOS adoption 
was higher in people aged 44 years and older [31]. While there have been instances where PMI's marketing strategy for IQOS was geared towards young people [32], we did not find evidence that Ploom TECH was marketed to an older age group. Interestingly, no significant associations were observed between gender, annual household income, or education level, and use of a particular HTP device brand. IQOS users had higher odds of using HTPs on a daily basis, while Ploom TECH users had higher odds of using HTPs less frequently (weekly or monthly). Taken together, IQOS and Ploom TECH users appeared to have distinct characteristics.

While the most common reason for choosing glo and Ploom TECH was "perceived reduction in health risk compared to smoking", it was "used by friend" for IQOS. This is a somewhat surprising finding, given that reduced risk claims are a major talking point for PMI's IQOS marketing [33]. The reduced risk claims are primarily based on a reduction in emission of numerous toxicants from HTPs as compared to combustible cigarettes. However, a review of industry documents has shown that some substances, such as propylene glycol, glycidol, acetol, and 2-propen-1-ol, are elevated compared to the combustible cigarette, due to the higher content of humectants in the tobacco filler of the HTP inserts [34]. With IQOS being the most popular HTP device brand and its odds of use higher among young adults, the peer use aspect as a motive for using IQOS is unsurprising as peer influence has been documented to influence young adults with respect to tobacco use behavior [35]. Additionally, "endorsement in the media" as a reason for choosing device brand was significantly higher for IQOS than for glo and Ploom TECH. This corresponds with observations from a previous study in Japan, which reported an exponential increase in internet searches for IQOS immediately following the airing of a single popular television episode, in which a Japanese comedian discussed his IQOS use [11]. Future studies should aim to examine whether these differences are substantiated as the market continues to evolve, and the degree to which individual product marketing and promotional strategies may contribute to users' reasons for adopting a particular HTP brand.

\subsection{Flavor Preference}

Regarding HTP flavor preferences, menthol was the most prevalent flavor preferred by current Japanese HTP users. Likewise, the popularity of menthol flavors in combustible cigarettes has been increasing in Japan, particularly among younger and female smokers [36]. Preference for menthol flavors among smokers has been reported to be inversely associated with age [37]. It is not surprising that HTP users, who are predominantly younger, also have preferences toward this flavor. The high popularity of menthol flavor among HTP users may have negative consequences for public health. Menthol flavor in cigarettes has been associated with increased initiation [37,38], greater nicotine dependence [39], lower quit rates [40], and higher relapse [38,39]. The tobacco industry has long utilized menthol flavor to attract users [39]. Given the wide variety of menthol flavors (and by extension other mentholated flavors, including mentholated fruity) being offered as tobacco-containing inserts, and what is known about the public health effects of menthol, the extensive use of menthol flavor for HTPs is of concern.

\subsection{Study Limitations}

There are several limitations to this study. First, data in this study were self-reported and we were not able to validate it, particularly when it comes to abstinence from cigarette smoking among exclusive HTP users. Second, this study was based on cross-sectional data; thus, causal inference should not be made. Third, even though ENDS is not commercially available in Japan, we did not evaluate the concurrent use of ENDS or ENNDS (electronic non-nicotine delivery system) among HTP users. Lastly, although the sampling and weighting strategies seek to ensure national representativeness, the possibility of excluding participants in a web-based survey cannot be ruled out. 


\section{Conclusions}

In 2018, the prevalence of current HTP use in Japan was 2.7\%, with most HTP users also smoking combustible cigarettes. The most popular brand device among HTP users was IQOS, followed by Ploom TECH and glo. Notable differences in age and frequency of HTP use were observed between IQOS and Ploom TECH users. Menthol was the most common flavor being used by HTP users. Given HTPs' significant presence, tobacco regulation in Japan should also include HTPs and should be tailored to specific groups of HTP users.

Author Contributions: Conceptualization, E.S. and M.L.G.; methodology, E.S. and D.M.S.; validation, E.S.; formal analysis, E.S.; investigation, I.Y., Y.M., T.T., J.O., A.C.K.Q., and G.T.F.; resources, I.Y., Y.M., T.T., S.X., J.O., A.C.K.Q., and G.T.F.; data curation, A.C.K.Q. and J.O.; writing-original draft preparation, E.S. and M.L.G.; writing-review and editing, C.M., R.J.O., D.M.S., K.M.C., A.H., T.T., A.C.K.Q., S.X., G.T.F.; visualization, E.S. and C.M.; supervision, D.S., M.L.G. and G.T.F.; project administration, A.C.K.Q., J.O., and G.T.F.; funding acquisition, G.T.F. and I.Y.

Funding: The 2018 ITC Japan Survey was supported by a grant from National Cancer Center and Research Development Fund (28-A-24), with additional support provided by Canadian Institutes of Health Research Foundation Grant (FDN-148477). G.T.F. was also supported by a Senior Investigator Award from the Ontario Institute for Cancer Research.

Conflicts of Interest: K.M.C. has received payment as a consultant to Pfizer, Inc., for service on an external advisory panel to assess ways to improve smoking cessation delivery in health care settings. K.M.C. also has served as paid expert witness in litigation filed against the tobacco industry. G.T.F. has served as an expert witness on behalf of governments in litigation involving the tobacco industry. M.L.G. has received a research grant from Pfizer and served as a member of scientific advisory board to Johnson \& Johnson. All other authors have no conflicts of interest to declare.

\section{Appendix A Survey Weighting for "Less than Weekly, but at Least Once a Month" HTP Users}

For survey weight, the definition for current HTP users in ITC Japan Survey is daily and weekly HTP users. Meanwhile, the definition for current HTP users in this study is daily, weekly, and monthly HTP users. Monthly HTP users were, therefore, eligible for the survey in exclusive smokers and non-users categories. As a result, they were given the survey weight in accordance with each respective group. The current setting implies that for weight calculation, monthly HTP users "behave" more like HTP non-users rather than like HTP users. Similarly, monthly HTP users who were also cigarette smokers "behave" more closely to exclusive smokers than to dual users.

The additions of monthly HTP users increased variability and thus reduce power for the regression analysis. This, however, is compensated by the increase in sample size coming from including monthly HTP users and inclusion of the frequency of HTP use as one of the covariates for the regression analysis.

\section{Appendix B}

Table A1. Flavor category for tobacco-containing insert products.

\begin{tabular}{cccc}
\hline No & Product Name & Device Brand & Flavor Category \\
\hline 1 & Marlboro Heatstick Balanced & IQOS & Tobacco \\
2 & Marlboro Heatstick Menthol & IQOS & Menthol \\
3 & Marlboro Heatstick Mint & IQOS & Menthol \\
4 & Marlboro Heatstick Purple Menthol & IQOS & Mentholated fruity \\
5 & Marlboro Heatstick Regular & IQOS & Tobacco \\
6 & Marlboro Heatstick Smooth & IQOS & Tobacco \\
7 & Kent Neostick Bright Tobacco & glo & Tobacco \\
8 & Kent Neostick Citrus Fresh * & glo & Mentholated fruity \\
9 & Kent Neostick Dark Fresh * & glo & Mentholated fruity \\
10 & Kent Neostick Fresh Mix & glo & Menthol \\
11 & Kent Neostick Intense Fresh & glo & Menthol \\
12 & Kent Neostick Refreshing Menthol & glo & Menthol \\
13 & Kent Neostick Regular & glo & Tobacco \\
\hline
\end{tabular}


Table A1. Cont.

\begin{tabular}{cccc}
\hline No & Product Name & Device Brand & Flavor Category \\
\hline 14 & Kent Neostick Rich Tobacco & glo & Tobacco \\
15 & Kent Neostick Smooth Fresh & glo & Menthol \\
16 & Kent Neostick Spark Fresh * & glo & Mentholated fruity \\
17 & Kent Neostick Strong Menthol & glo & Menthol \\
18 & Mevius for Ploom TECH Brown Aroma & Ploom TECH & Coffee \\
19 & Mevius for Ploom TECH Cooler Green & Ploom TECH & Mentholated fruity \\
20 & Mevius for Ploom TECH Cooler Purple & Ploom TECH & Mentholated fruity \\
21 & Mevius for Ploom TECH Red Cooler & Ploom TECH & Mentholated fruity \\
22 & Mevius for Ploom TECH Regular & Ploom TECH & Tobacco \\
\hline \multicolumn{2}{r}{${ }^{*}$ Product was only available in Miyagi Prefecture, but was shown to all applicable respondents. }
\end{tabular}

\section{References}

1. Brose, L.S.; Simonavicius, E.; Cheeseman, H. Awareness and Use of "Heat-not-burn" Tobacco Products in Great Britain. Tob. Regul. Sci. 2018, 4, 44-50. [CrossRef]

2. Simonavicius, E.; Mcneill, A.; Shahab, L.; Brose, L.S. Heat-not-burn tobacco products: A systematic literature review. Tob. Control 2019, 28, 582-594. [CrossRef] [PubMed]

3. World Health Organization. Heated tobacco products (HTPs) market monitoring information sheet. 2018. Available online: http://www.who.int/tobacco/publications/prod_regulation/heated-tobacco-products/en/ (accessed on 24 October 2019).

4. Bialous, S.A.; Glantz, S.A. Heated tobacco products: Another tobacco industry global strategy to slow progress in tobacco control. Tob. Control 2018, 27, s111-s117. [CrossRef] [PubMed]

5. Tabuchi, T.; Kiyohara, K.; Hoshino, T.; Bekki, K.; Inaba, Y.; Kunugita, N. Awareness and use of electronic cigarettes and heat-not-burn tobacco products in Japan. Addiction 2016, 111, 706-713. [CrossRef] [PubMed]

6. Kim, J.; Yu, H.; Lee, S.; Paek, Y.J. Awareness, experience and prevalence of heated tobacco product, IQOS, among young Korean adults. Tob. Control 2018, 27, s74-s77. [CrossRef]

7. Liu, X.; Lugo, A.; Spizzichino, L.; Tabuchi, T.; Pacifici, R.; Gallus, S. Heat-not-burn tobacco products: Concerns from the Italian experience. Tob. Control 2019, 28, 113-114. [CrossRef]

8. Nyman, A.L.; Weaver, S.R.; Popova, L.; Pechacek, T.F.; Huang, J.; Ashley, D.L.; Eriksen, M.P. Awareness and use of heated tobacco products among US adults, 2016-2017. Tob. Control 2018, 27, s55-s61. [CrossRef]

9. Marynak, K.L.; Wang, T.W.; King, B.A.; Agaku, I.T.; Reimels, E.A.; Graffunder, C.M. Awareness and Ever Use of "Heat-Not-Burn" Tobacco Products Among U.S. Adults, 2017. Am. J. Prev. Med. 2018, 55, 551-554. [CrossRef]

10. Tsugawa, Y.; Hashimoto, K.; Tabuchi, T.; Shibuya, K. What can Japan learn from tobacco control in the UK? Lancet 2017, 390, 933-934. [CrossRef]

11. Tabuchi, T.; Gallus, S.; Shinozaki, T.; Nakaya, T.; Kunugita, N.; Colwell, B. Heat-not-burn tobacco product use in Japan: Its prevalence, predictors and perceived symptoms from exposure to secondhand heat-not-burn tobacco aerosol. Tob. Control 2017, 27, e25-e33. [CrossRef]

12. Uranaka, T.; Ando, R. Philip Morris Aims to Revive Japan Sales with Cheaper Heat-Not-Burn Tobacco. Reuters. Tokyo. 2018. Available online: https://www.reuters.com/article/us-pmi-japan/philip-morris-aimsto-revive-japan-sales-with-cheaper-heat-not-burn-tobacco-idUSKCN1MX06E (accessed on 29 August 2019).

13. Uranaka, T.; Shimizu, R. Japan Tobacco to cut heated tobacco prices in battle with Philip Morris. Reuters. Tokyo. 2018. Available online: https://www.reuters.com/article/us-japan-tobacco/japan-tobacco-to-cutheated-tobacco-prices-in-battle-with-philip-morris-idUSKCN1IW0XH (accessed on 12 November 2019).

14. Kumamaru, H. Update of Current Status in Japan on Tobacco Harm Reduction. 2019. Available online: https://gfn.net.co/downloads/2019/presentations/Hiroya_Kumamaru.pdf (accessed on 12 November 2019).

15. Uranaka, T.; Geller, M. British American Tobacco to test tobacco e-cigarette in Japan. Reuters. Tokyo. 2016. Available online: https://www.reuters.com/article/us-brit-am-tobacco-ecigarettes-idUSKBN1330AG (accessed on 12 November 2019). 
16. Japan Tobacco Inc. JT Applies to Amend Retail Prices of Tobacco Products in Japan in Response to a Planned Excise Increase. 2018. Available online: https:/www.jt.com/media/news/2018/pdf/20180814_E01.pdf (accessed on 12 November 2019).

17. Sternbach, N.; Annunziata, K.; Fukuda, T.; Yirong, C.; Stankus, A. Smoking Trends in Japan From 2008-2017: Results From The National Health and Wellness Survey. Value Heal. 2018, 21, S105. [CrossRef]

18. Assunta, M.; Chapman, S. A “clean cigarette" for a clean nation: A case study of Salem Pianissimo in Japan. Tob. Control 2004, 13 (Suppl. 2), 58-62. [CrossRef] [PubMed]

19. McKelvey, K.; Popova, L.; Kim, M.; Chaffee, B.W.; Vijayaraghavan, M.; Ling, P.; Halpern-Felsher, B. Heated tobacco products likely appeal to adolescents and young adults. Tob. Control 2018, 27, s41-s47. [CrossRef] [PubMed]

20. Hair, E.C.; Bennett, M.; Sheen, E.; Cantrell, J.; Briggs, J.; Fenn, Z.; Vallone, D. Examining perceptions about IQOS heated tobacco product: Consumer studies in Japan and Switzerland. Tob. Control 2018, 27, s70-s73. [CrossRef] [PubMed]

21. Pollay, R.W. Targeting youth and concerned smokers: Evidence from Canadian tobacco industry documents. Tob. Control 2000, 9, 136-147. [CrossRef]

22. Wakefield, M.; Morley, C.; Horan, J.K.; Cummings, K.M. The cigarette pack as image: New evidence from tobacco industry documents. Tob. Control 2002, 11 (Suppl. 1), 73-80. [CrossRef]

23. Carpenter, C.M.; Wayne, G.F.; Pauly, J.L.; Koh, H.K.; Connolly, G.N. New cigarette brands with flavors that appeal to youth: Tobacco marketing strategies. Health Aff. 2005, 24, 1601-1610. [CrossRef]

24. Hafez, N.; Ling, P.M. Finding the Kool Mixx: How Brown and Williamson used music marketing to sell cigarettes. Tob. Control 2006, 15, 359-366. [CrossRef]

25. Cruz, T.B.; Wright, L.T.; Crawford, G. The menthol marketing mix: Targeted promotions for focus communities in the United States. Nicotine Tob. Res. 2010, 12 (Suppl. 2), 147-153. [CrossRef] [PubMed]

26. Ministry of Health, Labour and Welfare. Report on National Health and Nutritional Survey, Japan 2016. Ministry of Health, Labour and Welfare; 2018. Available online: http://www.mhlw.go.jp/bunya/kenkou/ eiyou/h28-houkou.html (accessed on 8 September 2019).

27. Japan Tobacco Inc. JT's Annual Survey Finds 21.1 Percent of Japanese Adults Are Smokers. 2018. Available online: https://www.jt.com/investors/media/press_releases/2012/0730_01.html (accessed on 1 September 2019).

28. Berry, K.M.; Fetterman, J.L.; Benjamin, E.J.; Bhatnagar, A.; Barrington-Trimis, J.L.; Leventhal, A.M.; Stokes, A. Association of Electronic Cigarette Use With Subsequent Initiation of Tobacco Cigarettes in US Youths. JAMA Netw. Open 2019, 2, e187794. [CrossRef] [PubMed]

29. Soneji, S.; Barrington-Trimis, J.L.; Wills, T.A.; Leventhal, A.M.; Unger, J.B.; Gibson, L.A.; Spindle, T.R. Association Between Initial Use of e-Cigarettes and Subsequent Cigarette Smoking Among Adolescents and Young Adults: A Systematic Review and Meta-analysis. JAMA Pediatr. 2017, 171, 788-797. [CrossRef] [PubMed]

30. Hwang, J.H.; Ryu, D.H.; Park, S. Heated tobacco products: Cigarette complements, not substitutes. Drug Alcohol Depend. 2019, 204, 107576. [CrossRef] [PubMed]

31. Roulet, S.; Chrea, C.; Kanitscheider, C.; Kallischnigg, G.; Magnani, P.; Weitkunat, R. Potential predictors of adoption of the Tobacco Heating System by U.S. adult smokers: An actual use study. F1000Res. 2019, 8, 214. [CrossRef] [PubMed]

32. Dyer, O. E-cigarette makers under fire for marketing to young people. BMJ 2019, 365, 2261. [CrossRef] [PubMed]

33. St Helen, G.; Jacob, P.; Nardone, N.; Benowitz, N.L. IQOS: Examination of Philip Morris International's claim of reduced exposure. Tob. Control 2018, 27 (Suppl. 1), s30-s36. [CrossRef] [PubMed]

34. Mallock, N.; Pieper, E.; Hutzler, C.; Henkler-Stephani, F.; Luch, A. Heated Tobacco Products: A Review of Current Knowledge and Initial Assessments. Front Public Health 2019, 7, 287. [CrossRef]

35. Freedman, K.S.; Nelson, N.M.; Feldman, L.L. Smoking initiation among young adults in the United States and Canada, 1998-2010: A systematic review. Prev. Chronic Dis. 2012, 9, E05. [CrossRef]

36. Connolly, G.N.; Behm, I.; Osaki, Y.; Wayne, G.F. The impact of menthol cigarettes on smoking initiation among non-smoking young females in Japan. Int. J. Environ. Res. Public Health 2011, 8, 1-14. [CrossRef] 
37. WHO Study Group on Tobacco Product Regulation (TobReg). Banning Menthol in Tobacco Products. World Health Organization. 2016. Available online: https://apps.who.int/iris/bitstream/handle/10665/205928/ 9789241510332_eng.pdf (accessed on 12 September 2019).

38. Lee, Y.O.; Glantz, S.A. Menthol: Putting the pieces together. Tob. Control 2011, 20 (Suppl. 2), 1-7. [CrossRef]

39. Villanti, A.C.; Collins, L.K.; Niaura, R.S.; Gagosian, S.Y.; Abrams, D.B. Menthol cigarettes and the public health standard: A systematic review. BMC Public Health 2017, 17, 1-13. [CrossRef]

40. Ahijevych, K.; Garrett, B.E. The role of menthol in cigarettes as a reinforcer of smoking behavior. Nicotine Tob. Res. 2010, 12 (Suppl. 2), 110-116. [CrossRef] [PubMed]

(C) 2019 by the authors. Licensee MDPI, Basel, Switzerland. This article is an open access article distributed under the terms and conditions of the Creative Commons Attribution (CC BY) license (http://creativecommons.org/licenses/by/4.0/). 